\title{
SIMPLIFIED THEORY OF THE HEAD-TAIL INSTABILITY OF COLLIDING BUNCHES
}

\author{
E. A. Perevedentsev, Budker Institute of Nuclear Physics, Novosibirsk, 630090, Russia
}

\section{Abstract}

A proposed theoretical model incorporates in an averaged form both the conventional head-tail effect in a single bunch (due to impedance elements in the machine), and the linear part of the coherent beam-beam interaction, with the account of the finite bunch length.

\section{INTRODUCTION}

For the strong head-tail effect in the beam-beam system we construct here an averaged version of the space-time domain formalism [1,2], aiming at clarity and solvability, at the sacrifice of localized interaction effects. This holds in situations when all the mode spectrum of interest lies far off the (half)integer tunes, i.e. for machines where the synchrotron tune and collective tuneshifts are much less than fractional betatron tune.

Applied to strong-strong collisions, the new theory predicts the coherent dipole beam-beam instability of head-tail type; estimates show that the chromaticity is effective in control of its increments.

\section{CIRCULANT EQUATIONS}

The main difficulty of multi-particle models in the spacetime domain is seen already in the educational 2-particle model in A. Chao's text [3]: the wake acts in alternating mode on either the 1st or the 2nd particle, resulting in timedependent coefficients in the equations of motion even with the constant wake.

The model employed in [2] and here is not based on real macroparticles which move longitudinally. It considers the synchrotron phase circle ${ }^{1}$ divided into $N$ fixed equal boxes with fixed longitudinal position in the bunch, see Fig. 1. These boxes are uniformly populated with particles each carrying its transverse dipole moment.

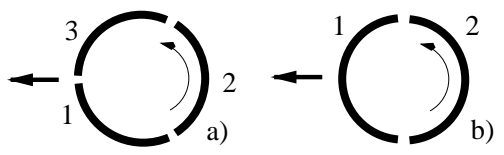

Figure 1: Division of the synchrotron phase into boxes, populated with particles; a) 3 divisions, CE3 formalism; b) 2 divisions for the CE2 formalism.

A variable dipole moment $d_{i}$ is ascribed to each $i$ th box, $i=1, \ldots, N$. The synchrotron oscillation just transports

\footnotetext{
${ }^{1}$ For the needs of this paper the hollow beam model will suffice, i.e. all the particles are assumed to have the same amplitude of the synchrotron oscillation.
}

the dipole moment around the circle, across the boxes, and we follow variations of $d_{i}$ in each box: no need to interchange the boxes when evaluating their interaction.

The dipole moments obey the betatron oscillation equation in the Courant-Snyder normalization, with the coupling in the RHS:

$$
\ddot{d}_{i}+\omega_{b}^{2} d_{i}=-2 \omega_{b} D_{i k} d_{k} .
$$

Here $\omega_{b}$ is the betatron frequency, the machine azimuth is used as quasi-time, and the dynamic matrix $D_{i k}$ approximates the integral linear operator of collective interaction. First of all we use the ansatz $d_{i}=a_{i} e^{-i \omega_{b} t}+$ c.c. and standard averaging to get rid of $a_{i}^{*}$ in the shortened equations:

$$
i \dot{a}_{i}=D_{i k} a_{k} .
$$

$N$ complex amplitudes $a_{i}$ of oscillating dipole moments sitting in each box, form a complete set of dynamic variables of the averaged problem. In fact, $a_{i}$ are sampled values of the continuous function of the synchrotron phase $\varphi$ : $a_{i}=a\left(\varphi_{i}, t\right)$, its total time derivative

$$
\dot{a}(\varphi, t)=\frac{\partial a}{\partial t}+\dot{\varphi} \frac{\partial a}{\partial \varphi}=\frac{\partial a}{\partial t}+\omega_{s} \frac{\partial a}{\partial \varphi}
$$

should be represented in our difference equations with a correct $N$-point approximation of $\partial a(\varphi, t) / \partial \varphi \rightarrow \gamma_{i k} a_{k}$. For periodic functions, $\gamma$ is known to be a special circulant matrix; we define another circulant $C=-i \omega_{s} \gamma$, and finally come from (2) to new equations

$$
i \dot{a}_{i}=\left(C_{i k}+D_{i k}\right) a_{k} .
$$

In what follows we will refer to Eqs. (3) as Circulant Equations, with the notation CE3 and CE2, for 3 and 2 boxes, respectively. Their remarcable feature is that the circulant matrix $C$ responsible for the free synchrobetatron motion, and the interaction matrix $D$ on the RHS are additive.

The synchrobetatron mode spectrum emerging from CE should first be checked for free oscillation. We start with $N=3$, Fig. 1a, and accordingly define the proper mode numbers $m$ as $-1,0,+1$. The 3 -point circulant is:

$$
C_{3}=\frac{i \omega_{s}}{\sqrt{3}}\left(\begin{array}{rrr}
0 & -1 & 1 \\
1 & 0 & -1 \\
-1 & 1 & 0
\end{array}\right) .
$$

Substituting for the proper modes $a_{i}(t)=v_{i} e^{-i \Omega t}$ in (3), with $D_{i k}=0$, we find the mode frequencies $\Omega$ and eigenvectors $v$ from the eigensystem of $C_{3}$ :

$$
\begin{aligned}
\Omega_{0} & =0, \quad v_{0}^{T}=\left(\begin{array}{cc}
1, & 1, \quad 1
\end{array}\right) ; \\
\Omega_{+1,-1} & = \pm \omega_{s}, v_{+1,-1}^{T}=\left(1, e^{ \pm 2 \pi i / 3}, e^{ \pm 4 \pi i / 3}\right)(5)
\end{aligned}
$$


Indeed, the free oscillation mode spectrum comprises the betatron frequency and two its synchrotron sidebands: $\omega_{b}, \omega_{b} \pm \omega_{s}$. Mind that all frequencies in the shortened equations are counted from $\omega_{b}$, e.g. those in (5). The mode eigenvectors $v$ give sampling of the Fourier harmonics $e^{i m \varphi}$, with $m=+1,0,-1$ (cf. [3], Eq. (6.185)), at 3 equidistant values of $\varphi_{k}$ according to division into 3 boxes.

In many cases coupling of only two modes in all the spectrum is important, those with the least separation in frequencies. $N=2$ boxes (Fig. 1b) then suffice to represent them. Coupling of these two modes to the other ones is neglected. Thus we come to the extremely simple circulant equation CE2, where free motion of the only two modes in question is given by (3) with $D=0$, and the $2 \times 2$ circulant:

$$
C=\frac{\omega_{s}}{2}\left(\begin{array}{rr}
-1 & 1 \\
1 & -1
\end{array}\right)
$$

Its eigensystem reads:

$$
\Omega_{0,-1}=0,-\omega_{s} ; \quad v_{0,-1}^{T}=(1, \quad \pm 1) .
$$

The mode spectrum here consists of the betatron frequency $\omega_{b}$ and only one sideband $\omega_{b}-\omega_{s}$ with exceptionally simple mode structure. It is convenient to take $\omega_{s}=1$ hereafter, i.e. to measure all the frequencies in the units of $\omega_{s}$.

\section{BEAM-BEAM MODES}

Now our task is to study the linear coherent dipole beambeam oscillations of longitudinally non-rigid bunches with finite length and incoherent synchrotron motion. We have to perform the same division into boxes in the both colliding bunches, like in Fig. 1, thus the set of variables $a_{i}$ in (2) is duplicated. All the matrices of the previous section have to be replaced accordingly, i.e.:

$$
C \rightarrow\left(\begin{array}{ll}
1 & 0 \\
0 & 1
\end{array}\right) \otimes C
$$

since they are relevant to each of the two bunches.

The beam-beam matrix $B$ (written for 2 boxes in each bunch) represents the linearized beam-beam force:

$$
B=-\frac{b I}{2}\left(\begin{array}{rrrr}
-2 & 0 & 1 & 1 \\
0 & -2 & 1 & 1 \\
1 & 1 & -2 & 0 \\
1 & 1 & 0 & -2
\end{array}\right)
$$

We consider here equal currents $I$ in each bunch, $b$ is the beam-beam tune slope in units of the synchrotron tune, $b I=\xi / Q_{s}, \xi$ is the conventional beam-beam parameter. Four proper modes, doubly degenerate, are given by the eigensystem of $C+B$ :

$$
\begin{aligned}
0 \pi) \Omega_{0 \pi} & =2 b I & v_{0 \pi}^{T}=(1,1,-1,-1), \\
0 \sigma) \Omega_{0 \sigma} & =0 & v_{0 \sigma}^{T}=(1,1,1,1), \\
-1 \sigma) \Omega_{-1 \sigma} & =b I-1 & v_{-1 \sigma}^{T}=(1,-1,1,-1), \\
-1 \pi) \Omega_{-1 \pi} & =b I-1 & v_{-1 \pi}^{T}=(1,-1,-1,1) .
\end{aligned}
$$

In the basis of these beam-beam modes all the matrices of Section 4 are blockwise diagonal: the interaction preserves orthogonality of the $\sigma$-modes subset to the subset of $\pi$ modes. Thus the characteristic equation of our 4-mode system always breaks up into a pair of quadratic equations, in CE2 formalism (a pair of cubuc equations in CE3), making the mode analysis so clear.

Finite bunch length $l$ of the order of the beta-function value $\beta^{*}$ at the IP, results in substantial betatron phase slippage over the interaction length. We include this effect in the phase lag parameter $y\left(0<y \approx l / 2 \beta^{*}<1\right)$, and modify the beam-beam matrix:

$$
B_{y}=\operatorname{diag}\left\{1, e^{2-i y}, 1, e^{-2 i y}\right\} \cdot B \cdot \operatorname{diag}\left\{1, e^{2 i y}, 1, e^{2 i y}\right\} .
$$

Eigenvalues of $C+B_{y}$ give the new mode tunes ${ }^{2}$, see (8) with $w=0$. The mode coupling results in repulsion between the mode tunes; they are always real and never merge. Thus, no instability can appear in this beam-beam model, unless we introduce some impedance elements.

\section{BEAM-BEAM INSTABILITY}

The impedance element(s) inevitably present in the machine act on each of the colliding bunches individually; in CE2 formalism they are plugged in the wake matrix $W$, e.g. for the constant wake ${ }^{3}$ we have:

$$
W=-\frac{w I}{2}\left(\begin{array}{ll}
1 & 0 \\
0 & 1
\end{array}\right) \otimes\left(\begin{array}{ll}
1 & 0 \\
2 & 1
\end{array}\right),
$$

$I$ is the beam current, $w$ is the coherent tune slope.

The chromaticity $\chi$ will cause the chromatic phase lag $2 x \propto-\chi$, (cf. [3], Eq. (4.88)), of the trailing box oscillation with respect to the leading one; we have to modify the wake matrix accordingly:

$W_{x}=\operatorname{diag}\left\{1, e^{-2 i x}, 1, e^{-2 i x}\right\} \cdot W \cdot \operatorname{diag}\left\{1, e^{2 i x} 1, e^{2 i x}\right\}$.

In combination with the beam-beam interaction, impedance elements completely change the situation. Consider first the case of very short bunches, $y \ll 1$. The mode tunes are eigenvalues of $C+B+W$, put $x=y=0$ in (8). The beam-beam tuneshift results in reduction of the modes $0 \sigma,-1 \sigma$ merge threshold: $(b+2 w) I_{t h}=1$, the closest one. The head-tail + beam-beam problem corresponding to this short bunch limit was studied numerically in the $2 \times 2$ particle model [4], predictions on possible reduction of the head-tail threshold due to beam-beam collisions and some of the results agree with ours.

The betatron phase slippage accounting for the finite bunch length in the beam-beam matrix $B_{y}$ gives a new instability, when acts in combination with wakes; we can see

\footnotetext{
${ }^{2}$ Saving the space, we omit here expressions for the mode tunes since they are available from the general formula (8) presented at the end of Section 4, being its particular cases.

${ }^{3}$ With a known wake function other than constant the coefficients in $W$ are available from the complete theory [2]. Or they may be considered as phenomenological coefficients, to be tuned to fit experimentally known head-tail parameters for a particular machine.
} 
this from the eigenvalues of $C+B_{y}+W$ (put $x=0$ in (8)). Simple analysis shows that modes $0 \sigma$ and $-1 \pi$ are unstable without a current threshold, as if in conventional head-tail instability, see Fig. 2, left. However, their increments $\delta$ start quadratically at low beam current: $\delta_{0 \sigma,-1 \pi}=\operatorname{Im} \Omega \approx b w I^{2} \sin y \cos y ; \delta_{-1 \sigma, 0 \pi}=-\delta_{0 \sigma,-1 \pi}$. Changing our normalized units to ordinary ones, we have: $\delta \rightarrow \omega_{s} \delta, b I \rightarrow \xi / Q_{s}, w I \rightarrow \Delta Q_{c o h} / Q_{s}$ and rewrite the increment via the revolution period $T_{0}$ :

$$
\delta \approx b w I^{2} \sin y \cos y \rightarrow 2 \pi \xi \Delta Q_{c o h} / T_{0} Q_{s} .
$$

One should take here $Q_{s}>\xi, \Delta Q_{c o h}$ large enough, to stay in the validity range of our 2-mode model. At high currents $\delta \sim \omega_{s}$ roughly, we deal with a fast instability.
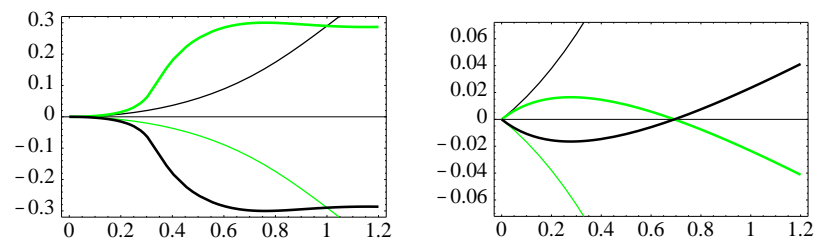

Figure 2: Effect of the positive chromaticity on the mode increments (plotted vs. the beam current) for combined action of the wake and betatron phase slippage in collision. Left: $x=0$, right: $x=-1.42$. Parameters in (8): $y=0.2$, $b=1.1, w=1$. All the $\sigma$ modes are shown in thick lines, $\pi$ modes in thin lines, the 0 modes in green, the -1 modes in black.

Physically, the phase slippage is somewhat similar to the chromatic phase effect. So, the chromaticity tuning gives us a possible cure of this instability. The chromatic phase lag $x$ is involved in both beam-beam and impedance action, and therefore the eigenvalues of $C+B_{x+y}+W_{x}$ are now needed:

$$
\begin{aligned}
\Omega_{0 \pi,-1 \pi}= & -\frac{1}{2}\left(1-(3 b-w) I \mp\left(1+b I e^{2 i(x+y)}\right)^{\frac{1}{2}}\right. \\
& \left.\times\left(1+\left(b e^{-2 i y}-2 w\right) I e^{-2 i x}\right)^{\frac{1}{2}}\right) \\
\Omega_{0 \sigma,-1 \sigma}= & -\frac{1}{2}\left(1-(b-w) I \mp\left(1-b I e^{2 i(x+y)}\right)^{\frac{1}{2}}\right. \\
& \left.\times\left(1-\left(b e^{-2 i y}+2 w\right) I e^{-2 i x}\right)^{\frac{1}{2}}\right)
\end{aligned}
$$

Due to zero sum of the increments, tuning chromaticity at a given phase slippage $y$ cannot stabilize all the modes, when $y \neq 0$. However, it strongly changes the increment partition: at positive chromaticities $(x<0)$ we can keep the increments of the both $\sigma$ modes reasonably small in a certain range of currents, see Fig. 2, right.

Fig. 3 shows how strongly absolute values of the decrements depend on the chromaticity knob; the optimum where the $\sigma$ and $\pi$ modes both have small decrements differs for the cases of shorter (Fig. 3, top) and longer (Fig. 3 , bottom) bunches, and varies with the current. An overall trend is that $x_{\text {opt }} \sim-1$, i.e. an elevated positive value of the chromaticity would help.
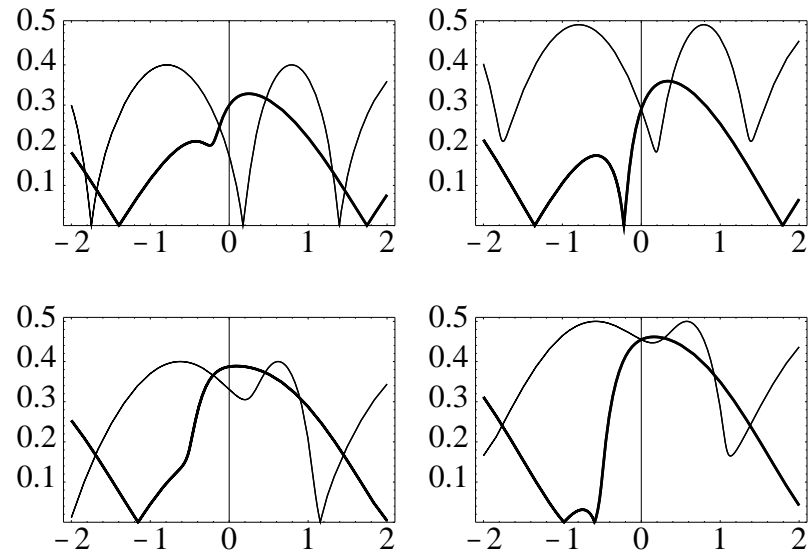

Figure 3: $|\operatorname{Im} \Omega|$ vs. the chromatic phase $x$ at two fixed beam currents $I$, left column: $I=0.8$, right column: $I=$ 1 ; for two bunch lengths $y$, top row: $y=0.2$, bottom row: $y=0.5$. Other parameters are the same as in Fig. 2. All the $\sigma$ modes are shown in thick lines, $\pi$ modes in thin lines.

\section{CONCLUSION}

A new formalism with Circulant Equations predicts stability of coherent dipole oscillations of non-rigid colliding bunches with finite length, in the linear beam-beam force model. Adding wakefields to the above strong-strong model, we find in such a combination a new beam-beam instability of head-tail type, arising without any current threshold. Its possible cure is the positive chromaticity.

Indeed, rather large positive chromaticities are known to help in improvement of the beam-beam performance on existing machines, e.g. VEPP-2M.

Any instability found in a linear theory is not necessarily lethal for a highly nonlinear beam-beam system. The amplitude growth is most likely to saturate at a certain level, but the onset of the instability may be a detriment to a low emittance regime.

Discussions with V.Parkhomchuk who has drawn my attention to the beam-beam problem with non-rigid bunches, are gratefully acknowledged.

\section{REFERENCES}

[1] V.V.Danilov and E.A.Perevedentsev, "Strong Head-Tail Effect and Decoupled Modes in the Spacetime Domain", in: Proc. 15th Int. Conf. on High Energy Accelerators, Hamburg, (1992), vol. 2, 1163.

[2] V.V.Danilov and E.A.Perevedentsev, "Feedback System for Elimination of the Transverse Mode Coupling Instability", CERN Report S1/93-38(AP), (1993).

V.V.Danilov and E.A.Perevedentsev, NIM, A 391, (1997), 77.

[3] A.W.Chao, Physics of Collective Beam Instabilities in High Energy Accelerators, (Wiley, 1993).

[4] K.Cornelis, "TMC Threshold as a Function of Beam-Beam Interaction”, CERN SL/Note 93-39(OP), (1993).

G.X.Li and K.Cornelis, "Calculation of TMC Threshold in the Presence of Beam-Beam Force", CERN Report SL/9485(AP), (1994). 